\title{
The Structure of the Stellar Hosts in Blue Compact Dwarf Galaxies
}

\author{
R.O. Amorín ${ }^{1}$, J.A.L. Aguerri ${ }^{1}$, L.M. Cairós ${ }^{2}$, N. Caon ${ }^{1}$, \\ and C. Muñoz-Tuñón ${ }^{1}$ \\ ${ }^{1}$ Instituto Astrofísico de Canarias, Vía Láctea s/n, E38200, La Laguna, Spain \\ email:ricardo.amorin@iac.es \\ ${ }^{2}$ Astrophysikalisches Institut Potsdam, An der Sternwarte 16, D-14482, Germany
}

\begin{abstract}
Blue compact dwarf (BCD) galaxies are gas-rich, low-luminosity $\left(M_{b} \gtrsim-18\right.$ mag) and compact systems, currently undergoing violent star-formation burst (Sargent \& Searle 1970). While it was initially hypothesized that they were very young galaxies (e.g. Sargent \& Searle 1970, et al. 1988), the subsecuent detection of an extended, redder stellar host galaxy showed that the vast majority of them are old systems (e.g. Gil de Paz et al. 2003,2005). BCDs play an important role for understanding the process of galaxy formation and evolution.

The structural properties of the low surface brightness stellar host in BCDs are often studied by fitting $r^{1 / n}$ models to the outer regions of their radial profiles. The limitations imposed by the presence of a large starburst emission overlapping the underlying component makes this kind of analysis a difficult task.

We propose a two-dimensional fitting methodology in order to improve the extraction of the structural parameters of the LSB host (Amorín et al. 2006, submitted). A set of ideal simulations are presented in order to test the reliability of the method and to determine its robustness and flexibility. We present the different steps of the method discussing its advantages and weaknesses. We compare the results for a sample of eight objects with those already obtained using a onedimensional technique (Caon et al. 2005).

We fit a PSF convolved Sérsic model to the $B V R$ images with the GALFIT publicly software (Peng et al. 2002). We restrict the fit to the stellar host by masking out the starburst region and take special care to minimize the sky-subtraction uncertainties. Consistency checks are performed to assess the reliability and accuracy of the derived structural parameters.

We obtain robust fits for all the sample galaxies, all of which, except one, show low Sérsic indices $n$-very close to 1 - with good agreement in the three bands. These findings suggest that the stellar hosts in BCDs have near-exponential profiles. Since the Sérsic index $n$ of host galaxies is important in the context of the possible structural and evolutionary connections among the different types of dwarf galaxies, we are currently extending the study to a larger sample of objects. This kind of studies will help us to understand the mechanisms that form and shape BCD galaxies, and how they relate to the other dwarf galaxy classes.
\end{abstract}

Keywords. galaxies: dwarf - galaxies: evolution - galaxies: photometry - galaxies: starburst galaxies: structure

\section{References}

Amorín, R. O., Muñoz-Tuñón, C., Aguerri, J. A. L., Cairós, L. M., \& Caon, N., 2006, A\&্A (Submitted).

Caon, N., Cairós, L. M., Aguerri, J. A. L., \& Muñoz-Tuñón, C. 2005, ApJS, 157, 218.

Kunth, D., Maurogordato, S., \& Vigroux, L. 1988, A\&GA, 204, 10.

Gil de Paz, A., Madore, B. F., \& Pevunova, O. 2003, ApJS, 147, 29.

Gil de Paz, A., \& Madore, B. F. 2005, ApJS, 156, 345.

Peng, C. Y., Ho, L. C., Impey, C. D., \& Rix, H.-W. 2002, AJ, 124, 266.

Sargent, W. L. W. \& Searle, L. 1970, ApJ, 162, L155. 